\title{
THE FEFFERMAN-STEIN TYPE INEQUALITIES FOR THE MULTILINEAR STRONG MAXIMAL FUNCTIONS
}

\author{
JuAn Zhang, Hiroki SAito And Qingying Xue
}

Abstract. Let $\vec{\omega}=\left(\omega_{1}, \ldots, \omega_{m}\right)$ be a multiple weight and $\left\{\Psi_{j}\right\}_{j=1}^{m}$ be a sequence of Young functions. Let $\mathscr{M}_{\mathscr{R}}^{\vec{\Psi}}$ be the multilinear strong maximal function with Orlicz norms which is defined by

$$
\mathscr{M}_{\mathscr{R}}^{\vec{\Psi}}(\vec{f})(x)=\sup _{R \in \mathscr{R}, R \ni x} \prod_{j=1}^{m}\left\|f_{j}\right\|_{\Psi_{j}, R},
$$

where the supremum is taken over all rectangles with sides parallel to the coordinate axes. If $\Psi_{j}(t)=t$, then $\mathscr{M}_{\mathscr{R}}^{\vec{t}}$ coincides with the multilinear strong maximal function $\mathscr{M}_{\mathscr{R}}$ defined and studied by Grafakos et al. In this paper, we first investigated the Fefferman-Stein type inequality for $\mathscr{M}_{\mathscr{R}}^{\vec{\Psi}}$ when $\vec{\omega}$ satisfies the $A_{\infty, \mathscr{R}}$ condition. Then, for arbitrary $\vec{\omega} \geqslant 0\left(\right.$ each $\left.\omega_{j} \geqslant 0\right)$, the Fefferman-Stein type inequality for the multilinear strong maximal function $\mathscr{M}_{\mathscr{R}}$ associated with rectangles will be given.

Mathematics subject classification (2010): 42B20, 47G10.

Keywords and phrases: Multilinear strong maximal function, Fefferman-Stein type inequality, Young function, multiple weights.

\section{REFERENCES}

[1] R. J. BAGBY, D. S. KURTZ, $L(\log L)$ spaces and weights for the strong maximal function, J. Anal. Math. 44 (1984/1985), 21-31.

[2] O. N. CAPRI, C. E. GUTIÉRREZ, Weighted inequalities for a vector-valued strong maximal function, Rocky Mountain J. Math. 18 (1988), 565-570.

[3] N. FAVA, Weak type inequalities for product operators, Studia Math. 42, 3 (1972), 271-288.

[4] N. FAVA AND O. N. CAPRI, Strong differentiability with respect to product measures, Studia Math. 78, 2 (1984), 173-178.

[5] A. CóRdoba, AND R. FEFFERMAn, A geometric proof of the strong maximal theorem, Ann. of Math. (2) 102, 1 (1975), 95-100.

[6] C. Fefferman And E. M. Stein, Some maximal inequalities, Amer. J. math. 93, 1 (1971), 107-115.

[7] L. Grafakos, L. Liu, C. Pérez, R. H. Torres, The Multilinear Strong Maximal Function, J. Geom. Anal. 21 (2011), 118-149.

[8] P. A. Hagelstein, T. LuQue, And I. Parissis, Tauberian conditions, Muckenhoupt weights, and differentiation properties of weighted bases, Trans. Amer. Math. Soc. 367, 11 (2015), 7999-8032.

[9] P. A. HAgelstein, AND I. PARISSis, The endpoint Fefferman-Stein inequality for the strong maximal function, J. Funct. Anal. 266, 1 (2014), 199-212.

[10] B. JAWERTH, Weighted inequalities for maximal operators: linearization, localization, and factorization, Amer. J. Math. 108 (1986), 361-414.

[11] B. Jessen, J. Marcinkiewicz, And A. Zygmund, Note on the differentiability of multiple integrals, Fund. Math. 25 (1935), 217-234.

[12] L. LiU And T. LuQue, $A B_{p}$ condition for the strong maximal function, Trans. Amer. Math. Soc. 366, 11 (2014), 5707-5726. 
[13] A. K. Lerner, S. Ombros, C. Pérez, R. H. Torres, R. Trujillo-González, New maximal functions and multiple weights for the multilinear Calderón-Zygmund theory, Adv. in Math. 220, 4 (2009), 1222-1264.

[14] T. LuQUE AND I. PARISSIS, The endpoint Fefferman-Stein inequality for the strong maximal function, J. Funct. Anal. 266, 1 (2014), 199-212.

[15] T. Mitsis, The weighted weak type inequality for the strong maximal function, J. Fourier Anal. Appl. 12, 6 (2006), 645-652.

[16] H. SAITO, AND H. TANAKA, The Fefferman-Stein type inequality for strong and directional maximal operators in the plane, arXiv: 1610.03186v1.

[17] H. TANAKA, The Fefferman-Stein type inequality for strong maximal operators in the higher dimensions, arXiv: $1611.01252 \mathrm{v} 2$. 